\title{
Reseña:
}

\section{Neves, I. S. V., Corradi, W., \& Castro, C. L. F. (2016). Ead: diálogos, compartilhamentos, práticas e saberes. Belo Horizonte: Universidade do Estado de Minas Gerais.}

\begin{abstract}
Maribel Deicy Villota ${ }^{1}$, Jakeline Amparo Villota Enríquez ${ }^{2}$, Efraín Bámaca López ${ }^{3}$
${ }^{1}$ Universidade Federal de São Carlos - UFSCAR. Programa de Pós-Graduação em Ciência, Tecnologia e Sociedade. Rodovia Washington Luís, km 235, São Carlos - SP, Brasil. mares-696@ hotmail.com. ${ }^{2}$ Universidad Santiago de Cali - USC. ${ }^{3}$ Universidade Federal de São Carlos - UFSCAR
\end{abstract}

Este texto presenta un sumario de artículos y relatos que abordan los diálogos y prácticas pedagógicas a través de la educación a distancia - EaD - en Brasil, buscando acrecentar la oferta de esta modalidad, como una estrategia para atender las demandas de formación en cuanto al desenvolvimiento y la vinculación de programas de enseñanza a distancia, en Instituciones Públicas. En Brasil, el Marco Legal para a Educación a Distancia, está contenido en el artículo 80 de la Ley de Directrices y Bases de la Educación Nacional $\mathrm{n}^{\circ}$ 9394/96, especificando que el Poder Público, incentivará el desenvolvimiento y la vinculación de enseñanza de programas a distancia. De esta manera, el objetivo de este texto es impulsar la $\mathrm{EaD}$, como estrategia para atender a la población, generando una discusión importante entorno a la comprensión holística de esa modalidad, entendiendo que existen desafíos, limites, posibilidades académicas y demás características aún por superar.

Así, el libro se divide en dos partes: la primera se compone de doce artículos resultante de las conferencias dadas en el Seminário Diálogos sobre EaD: Práticas pedagógicas, organizado por El Centro de Investigación en Educación a Distancia (CEPEAD) de la Universidad del Estado de Minas Gerais (UEMG) y el Simposio Internacional - Compartiendo prácticas y saberes en EaD: IES Públicas Brasileras y la Universidad Aberta de Portugal UAb, organizado por el Centro de Educación Abierta y a Distancia de la Universidade 
Federal de Ouro Preto (UFOP). La producción intelectual de esta primera parte está compuesta de artículos, resultados de las conferencias de ambos eventos, y la segunda, la presentación de diez relatos y experiencias exhibidas en el transcurso de estos eventos, enriqueciendo el debate sobre cuestiones teóricas y empíricas que hace pensar la $\mathrm{EaD}$ con parámetros de calidad al alcance de los ciudadanos.

En la primera parte, se presenta una conceptualización sobre la $\mathrm{EaD}$, el concepto de la etimología de la palabra, el análisis de la importancia de las prácticas pedagógicas, el uso del material didáctico utilizado en los cursos a distancia, los abordajes teórico-metodológicos, la utilización de las TIC's, y la posibilidad de acciones de laboratorio de poéticas fronterizas en el campo de la ciencia y la tecnología. El primer artículo Diálogos sobre EaD y prácticas pedagógicas de Inajara de Salles, remete a pensar los procesos educativos bajo una perspectiva planteada del mundo contemporáneo, quien legitima una lógica de tiempo, volcada a valorizar el hacer, es decir una forma particular de construir conocimiento y avanzar en la sociedad. Entender este desafío es importante, porque la construcción emergente de la $\mathrm{EaD}$ es relativo a las políticas públicas de iniciación científica, extensión, permanencia, suceso y aspectos normativos.

Seguido, encontramos el artículo Importancia del material didáctico para la educación a distancia de Cynthia Braga, quien enfatiza sobre la diferenciación del material didáctico de $\mathrm{EaD}$ respecto del material impreso tradicional. Este soporte hace parte de los recursos tecnológicos que pueden ser empleados durante la formación, entre ellos se destacan: a) autoinstitucional, solo informativos; b) movile learning, utilizan comunicación vía celular; c) imersivo, utilizan software; y d) colaborativo/cooperativo, utilizan ambientes virtuales AVA o también denominados SGC, sistema de gerenciamiento de recurso. Estos elementos permiten la vinculación de un contenido que envuelve a alumnos, profesores y tutores, en una dinámica de interfaz en el que se da una relación pedagógica a pesar de no estar físicamente envueltos en un diálogo.

Los artículos tres y cuatro: $L a$ investigación en la educación a distancia: Una mirada sobre los abordajes teóricometodológico de las pesquisas en EaD presentadas en la ANPED/2011-2013, y Las TICs como estrategia pedagógica para a educación, vinculan la modalidad histórica de la trayectoria de la $\mathrm{EaD}$, como 
modalidad de enseñanza desde los siglos XVII - XVIII, donde a partir de 1970 y finales de 1990, la $\mathrm{EaD}$ se torna un marco importante en la educación académica Brasilera, con garantías legales. Dentro de las primeras formas de $\mathrm{EaD}$, se destacan los programas de $\mathrm{TV}$, quienes pasaron a emitir posteriormente programas de educación. Así, los avances digitales permitieron el acceso a las tecnologías comunicativas, telemática, redes visuales, tecnologías de la información y comunicación, permitiendo innovar en los procesos educacionales.

La discusión de los proyectos de investigación en el Laboratorio de Poéticas Fronterizas (LABFRONT), también se aborda por Pablo Govira en su texto Del mundo codificado al mundo gamificado: posibilidades de las tecnologías digitales para la enseñanza a distancia, quien a través de un estudio transversal, propone problematizar las fronteras de la construcción de saberes que acontecen en la interface de enseñanza y extensión. Esto es importante porque permite instaurar un dialogo con los avances digitales, estableciendo una interacción hombremáquina frente a las TICs, tema abordado en el capítulo seis por Priscila Ramos a través de su texto Competencias del tutor y andrología: conceptos, principios y aplicaciones, modalidad reciente de la educación ocurrida a partir de los avances digitales de 1990, donde la $\mathrm{EaD}$ es vista como una actividad modeladora de unión entre las tecnologías y el hombre, vinculando a los estudiantes y profesores en torno a una ciberespacio, conectando ambientes variados en red.

Los textos siete, ocho y nueve, Estadio supervisado en cursos de licenciaturas presenciales y a distancia, La importancia estratégica de las universidades y de la educación a distancia $y$ en red en el siglo XXI, y Programa Nacional Escuela de Gestores de la educación básica pública, dimensionan la $\mathrm{EaD}$ como un estadio curricular surgido en el marco legal de obligatoriedad. Sin embargo, se analizan también referentes como la organización y el funcionamiento de las mejores universidades del mundo, frente al tema del $\mathrm{EaD}$; aprendizaje online destinado a personas de todo el mundo y perfilado actualmente por la Unión Europea y los gobiernos de los Estados Europeos en vistas a un cambio en la educación y la internacionalización. Bajo estos referentes, la UFMG describe como el curso ofertado por la Facultad de Educación fue pionero en la modalidad de $\mathrm{EaD}$, enfrentado resistencias y contradicciones percibidas bajo la noción de la EaD como de baja calidad, frente a los intereses del mercado. 
Un décimo artículo Políticas públicas en EaD en Brasil de Eucidio Arruda, complementa la inmersión de las tecnologías en busca de la comprensión de los procesos educativos; una mirada dirigida hacia la transitoriedad de las tecnologías, frente a las modificaciones pedagógicas y organizacionales, en la construcción de una composición formativa más contemporánea. Este proceso organizativo, genera cuestión al interior de la construcción de los procesos EaD sobre: ¿Qué es un gestor? ¿Qué se debe saber? ¿Cómo se compone la gestión? ¿La gestión de la $\mathrm{EaD}$ es similar a la gestión tradicional? ¿Qué particularidades pueden ser indicadas para la gestión de la educación a distancia virtual? ¿Qué aspectos un buen gestor debe gerenciar? Temática abordada en el undécimo artículo Gestión estratégica de la educación a distancia: constitución, complejidad y desafíos de Daniel Mill.

Cerrando la primera parte se ofrece: La educación a distancia en la Universidade Federal de ouro preto UFOP: panorama e expectativas de Inajara de Salles y Helton Cristian de Paula, quienes realizan un histórico institucional y experiencial de la $\mathrm{EaD}$ en los diversos proyectos implementados por la institución, este análisis se extiende hasta IES - Instituciones de Educación
Superior - públicas en donde la política de gobierno escogida provee grandes avances para esta modalidad.

La segunda parte del libro está compuesta por relatos y hechos relacionados con la experiencia directa del EaD. El primer texto Percepção de um coordenador de polo de apoio presencial sobre o desenvolvimento da UAB de Márcio Santos, resalta la importancia los coordinadores de polo frente al apoyo presencial destacándose el SisUAB, plataforma de soporte para el acompañamiento y la gestión de la Universidad Abierta en el Brasil. La siguiente intervención Tutoria a distância do curso da pedagogia da UAB/UEMG de Renata Mayra de Castro, relata la tensión entre el tiempo disponible de los tutores y el contenido del programa que exige un vínculo con la red pública, función ejercida sobre todo por profesores extremadamente cargados, puesto que la docencia en red se vuelve cada vez más desafiadora.

Se destacan también textos como Desafíos y experiencias de la EaD/UEMG de Alan Fagundes, con el actual contexto $\mathrm{EaD}$ en UEMG cuya preocupación genera un desafío frente a la calidad de los procesos de enseñanza y aprendizaje. De igual importancia en la misma perspectiva los textos: La experiencia del curso de libras a distancia en la UEMG, 
Villota, M. D., Enríquez, J. A. V., \& López, E. B. (2017). Reseña del texto: Neves, I. S. V., Corradi, W., \& Castro, C. L. F. (2016). Ead: diálogos, compartilhamentos, práticas e saberes...

presentando una organización y sistematización de datos de una cuesta online para 903 alumnos. El texto Las prácticas pedagógicas en el curso de pedagogía a distancia/UAB de la UEMG, presenta las practicas pedagógicas colocadas en un foro en relación a la identidad académica.

Se Destacan también: Estrategias y recursos formativos en la modalidad a distancia, quien asume el proyecto de formación continuada de gestores educacionales con formación de calidad; La evaluación y la relación pedagógica en la tesitura de los foros de discusión, acerca de los ambientes virtuales como foros de discusión y prácticas evaluativas coherentes en relación con la enseñanza; Salas virtuales de coordinación de AVA MOODLE, pesquisa realizada en torno a las salas virtuales como espacios utilizados por docentes; La utilización de representaciones táctiles en curso de formación de profesores, frente a la búsqueda de recursos didácticos para el desenvolviendo de las prácticas inclusivas para el mejoramiento de la EaD; Los Modelos de organización de la tutoría en $E a D$, presenta una compensación de la tutoría como estructura de trabajo en relación con los alumnos y profesores en busca de la calidad; y La vieja cuestión de los mitos y verdades de la EaD, y La realización de las polaridades, reflexiones basadas en las experiencias $\mathrm{EaD}$ en medio de limitaciones y respuestas en construcción en busca de un mejor modelo de actuación.

Todos los anteriores, representando una crítica a la experiencia de la percepción de la $\mathrm{EaD}$ como un desafío importante frente a las prácticas pedagógicas públicas presenciales, en constante tensión en medio de la calidad, la modalidad, la intencionalidad, la formación, la interacción, entre otros elementos. Dicho debate enriquece la temática de la $\mathrm{EaD}$ en la medida que problematiza los desafíos, límites, posibilidades y voluntades académicas frente al avance de las nuevas tecnologías, que permiten vislumbrar cada vez más de cerca la enseñanza y el aprendizaje a distancia, como herramienta útil para algunas poblaciones. Una idea conjuga todo el texto: se debe pensar en la $\mathrm{EaD}$, como un parámetro de calidad que divisa los alcances y los objetivos de la formación académica de un determinado grupo, como una oportunidad estratégica en comunidades carentes de educación presencial, problema presente en el diario vivir de una gran mayoría de personas en el mundo. 
Villota, M. D., Enríquez, J. A. V., \& López, E. B. (2017). Reseña del texto: Neves, I. S. V., Corradi, W., \& Castro, C. L. F. (2016). Ead: diálogos, compartilhamentos, práticas e saberes...

\section{Referencia}

Neves, I. S. V., Corradi, W., \& Castro, C. L. F. (Orgs.). (2016). Ead: diálogos, compartilhamentos, práticas e saberes. Belo Horizonte: Universidade do Estado de Minas Gerais.

Recebido em: 20/01/2017

Aprovado em: 20/02/2017

Publicado em: 29/06/2017

Como citar este artigo / How to cite this article / Como citar este artículo:

APA:

Villota, M. D., Enríquez, J. A. V., \& López, E. B. (2017). Reseña del texto: Neves, I. S. V., Corradi, W., \& Castro, C. L. F. (2016). Ead: diálogos, compartilhamentos, práticas e saberes. Belo Horizonte: Universidade do Estado de Minas Gerais. Rev. Bras. Educ. Camp., 2(1), 436-441. DOI: http://dx.doi.org/10.20873/uft.2525-

$\underline{4863.2017 \mathrm{v} 2 \mathrm{n} 1 \mathrm{p} 436}$

ABNT:

VILLOTA, M. D.; ENRÍQUEZ, J. A. V.; LÓPEZ, E. B. Reseña del texto: NEVES, I. S. V.; CORRADI, W.; CASTRO, C. L. F. Ead: diálogos, compartilhamentos, práticas e saberes. Belo Horizonte: Universidade do Estado de Minas Gerais, 2016. Rev. Bras. Educ. Camp., Tocantinópolis, v. 2, n. 1, p. 436-441, 2017. DOI: http://dx.doi.org/10.20873/uft.25254863.2017v2n1p436 